\title{
The effects of adrenal hormones on brain and body size
}

\author{
L. D. DEVENPORT and J. A. DEVENPORT \\ University of Oklahoma, Norman, Oklahoma
}

\begin{abstract}
By surgical means and selective hormone replacement, the adrenal secretions responsible for the suppression of brain growth were investigated. Adrenalectomy, but not demedullation, promoted brain growth, indicating that the active hormone was a cortical steroid. Replacement at various doses of mineralocorticoids (aldosterone, .02 and $.5 \mathrm{mg} / \mathrm{kg}$; deoxycorticosterone, 1.0 and $3.0 \mathrm{mg} / \mathrm{kg}$ ) and corticosterone $(1,3,15 \mathrm{mg} / \mathrm{kg})$ revealed that mineralocorticoids were without influence on hindbrain weight, forebrain weight, or linear dimensions. These hormones did, however, exact a pronounced effect on body weight, seeming to increase fat deposition in a dosedependent manner. They did not, however, affect skeletal growth as assessed by body (nose to anus) length. Corticosterone strongly depressed brain weights and dimensions, indicating that this glucocorticoid was the likely steriod whose removal by adrenalectomy augmented brain size. Corticosterone also decreased body weight, seemingly at the expense of fat stores, since it had no effect on body length. A combined dose of corticosterone $(8 \mathrm{mg} / \mathrm{kg})$ and deoxycorticosterone $(.6 \mathrm{mg} / \mathrm{kg}$ ) administered to adrenalectomized rats was found to very closely match the brain and body characteristics of intact rats.
\end{abstract}

Although adrenal cortical steroids have been firmly implicated in the regulation of blood sugar and sodium-hence the designations gluco- and mineralocorticoids-their actions are, in fact, considerably more diverse than this. Glucocorticoids are now known to bind to brain, especially limbic, tissue where they apparently alter neural activity (Pfaff, Silva, \& Weiss, 1971). These findings lent support to the already accumulating clinical (e.g., Quarton, Clark, Cobb, \& Bauer, 1955) and experimental (e.g., Di Giusto, Cairncross, \& King, 1971) evidence for personality and behavioral changes attendant on glucocorticoid administration.

Distinct from functional changes brought about by adrenal steroids, microscopic structural effects are beginning to be described. High adrenocortical steroid levels are linked with CNS cellular changes accompanying senescence (Landfield, Baskin, \& Pitler, 1981; Landfield, Waymire, \& Lynch, 1978), altered cerebellar tissue proliferation (Bohn \& Lauder, 1978), and reduced axonal sprouting in adult animals (Scheff, Bernardo, \& Cotman, 1980).

Although a variety of physiological manipulations, for example, starvation, are known to simultaneously change brain and body growth, only adrenalectomy has been shown to selectively influence the gross structure of the brain (Devenport, 1979). Ad-

We wish to acknowledge Organon, Oss, Holland, for their generous gift of aldosterone. We thank David Kem, University of Oklahoma Health Sciences Center, for the radioimmunoassay work. Our mailing address is: Department of Psychology, University of Oklahoma, Norman, Oklahoma 73019. renal extirpation in male rats increases forebrain and hindbrain growth by 6\%-10\% (wet and dry weight) in 30 days. This finding has since been replicated (Meyer, 1981) and extended to suggest that at least some of the growth is attributable to oligodendroglia proliferation.

In the present series of experiments, we attempt to more thoroughly specify the active hormones, to describe the gross brain and body changes, and to extend the findings to female rats.

\section{EXPERIMENT 1}

\section{Method}

Subjects. Female Sprague-Dawley rats were reared in our laboratory. Following sperm-positive vaginal smears, their dams were housed in individual wire-topped polyethylene cages. Within $12 \mathrm{~h}$ of birth, litters were sexed, reduced to 10 pups, and left undisturbed except for food and water maintenance. Litters were weaned at 23 days of age. The animals were group-housed, three per single hanging steel cage, until surgery at 25 days. All experimental groups to be described comprised female pups randomly derived from at least five litters.

Surgery and Maintenance. Under ether anesthesia, preceded by atropine sulfate $(.2 \mathrm{mg} / \mathrm{kg})$, adrenal glands were approached dorsally through small $(1-\mathrm{cm})$ bilateral incisions. For adrenalectomized (ADX) animals, each adrenal in its entirety was removed $(n=20)$. In the case of demedullated (DM) rats, the gland was exposed in situ, opened, and gently squeezed from beneath, the extruded medulla was recovered and removed $(n=8)$. The surgical procedure for sham-operated rats (SHAMS) was the same as that for ADXs and DMs, except that the adrenals were exposed and manipulated but left intact $(n=17)$. The body-wall incision was closed with surgical gut, the skin by wound clips. The rats were permitted 1-2 $\mathrm{h}$ in a heated recovery unit before being returned to their former cages. The following day, each was housed with a rat from the same group and given a continuous supply of isotonic 
saline (see Devenport, 1973, for preparation) and Purina Lab Chow. At 30 days of age, all were individually housed for the remainder of the experiment. In order to maintain comparability with subsequent experiments, beginning at 27 days of age, the rats were injected daily at $1500 \mathrm{~h}$ with $.1-.2$ cc sesame oil subcutaneously, based on body weight which was determined every fourth day. This occurred $1 \mathrm{~h}$ before onset of the dark phase of a LD 12:12 light cycle. If, over the course of weeks, an accumulation of subcutaneous oil was noticed, it was aspirated via a 20-ga needle.

Other female littermates underwent identical housing conditions but experienced no surgical procedures. These unoperated animals (UNOP) were not handled, injected, or otherwise disturbed $(n=15)$. Their body weights were recorded only at the beginning and end of the experiment.

Brain and body measurements. At 65 days of age, the rats were sacrificed with sodium pentobarbital overdose. Their body weights and body length (nose to anus) were measured, and approximately $5 \mathrm{cc}$ of blood was taken by exsanguination before perfusing intraaortically with a modified "instant processing" (Bondonna, Jacquet, \& Wolf, 1977) Formalin solution. The serum portion of the blood was drawn off and frozen after $15 \mathrm{~min}$ of centrifugation.

Brains were removed, taking care to preserve such easily damaged features as the cercbellar parafloccular lobes, optic chiasm, and olfactory bulbs. Once removed, the brain was prepared as previously described (Devenport, 1979). Briefly, this included the separation of the brain into fore- and hindbrain components by an intercollicular cut that emerged ventrally at the exact border of the pons and midbrain. The hindbrain, for our purposes, comprised all structures extending posterior to this cut and ended at the decussation of the pyramids. Cranial nerves were cut even with the surface from which they emerged, with the exception of the optic tracts and chiasm, which were retained. The optic nerves were removed and the olfactory bulbs were trimmed flush with the frontal pole of the brain. The brains were stored in "instant processing" Formalin for $24 \mathrm{~h}$ before being blotted with gauze and weighed on an analytical balance to the nearest $.1 \mathrm{mg}$. Brain material was weighed without knowledge of its origin.

The linear dimensions of the forebrain portions were measured with precision calipers to the nearest $.01 \mathrm{~cm}$. Measurements were taken for length, width, and depth at their maxima.

\section{Results}

Autopsy revealed no regenerated tissue among ADXs. DMs displayed obvious evidence of adrenal cortical tissue repair and proliferation, but their glands were without a medullar core.

Analysis of variance yielded significant group differences for every brain measurement $[\mathrm{Fs}(3,56)=$ 5.75-23.58, ps $<.0001-.002]$. As for body measurements, weight, not length, was significant $[F(3,56)$
$=12.66, \mathrm{p}<.0001 \mathrm{]}$, and body weight was inversely correlated with hindbrain and forebrain weight measurements [rs $=-.38$ to -.40 , ps $<.002-.003$ ], indicating that animals with large brains tended to have small bodies.

As can be seen in Table 1, it was the ADX group that was unique: Individual comparisons (Duncan's multiple range test) between rows in Table 1 found that ADXs differed significantly $(\alpha=.05)$ from all other groups across forebrain and hindbrain weight, and forebrain length, width, and depth. The DMs, UNOPs, and SHAMs did not differ among themselves.

Body length did not differ among the groups. The body weight of DMs was significantly greater than that of all other groups, and ADXs weighed significantly less. Taken together, the differences in body weight in the face of similar body lengths can probably be ascribed to variation in body fat deposition, an inference in keeping with subjective impressions gained during autopsy.

\section{Discussion}

These findings demonstrate that the adrenal factorwhatever it is-responsible for suppressing gross brain growth is present in females and must be a product of the adrenal cortex and not the medulla, the brains of DM rats not differing from SHAMs or UNOPs.

We are also permitted to conclude that the adrenalectomy-induced increase in brain weight is somewhat evenly distributed across the CNS. Forebrain and hindbrain weights were similarly affected by adrenalectomy, and the cerebrum was expanded in every direction.

That the differences in brain size are not merely owing to overall accelerated growth in ADXs is plainly revealed in the significant negative correlation between brain size and body weight; adrenalectomized animals carried heavier brains in lighter bodies. We found no reason to conclude that their skeletal growth was retarded; rather, adipose tissue may have been diminished.

Table 1

Surgical Effects on Mean Brain and Body Size

\begin{tabular}{|c|c|c|c|c|c|c|c|c|c|c|c|c|c|c|c|}
\hline \multirow{3}{*}{$\begin{array}{l}\text { Sur- } \\
\text { gery }\end{array}$} & \multicolumn{6}{|c|}{ Weight } & \multicolumn{8}{|c|}{ Linear Dimensions } & \multirow[b]{3}{*}{$\mathrm{n}$} \\
\hline & \multicolumn{2}{|c|}{ FB } & \multicolumn{2}{|c|}{ HB } & \multicolumn{2}{|c|}{ Body } & \multicolumn{2}{|c|}{ FB-L } & \multicolumn{2}{|c|}{ FB-W } & \multicolumn{2}{|c|}{ FB-D } & \multicolumn{2}{|c|}{ Body } & \\
\hline & Mean & SEM & Mean & SEM & Mean & SEM & Mean & SEM & Mean & SEM & Mean & SEM & Mean & SEM & \\
\hline ADX & 1302.86 & 14.42 & 478.16 & 8.47 & 205.14 & 4.39 & 1.46 & .01 & 1.46 & .01 & .96 & .01 & 20.18 & .21 & 20 \\
\hline SHAM & 1179.68 & 16.36 & 439.29 & 4.91 & 237.79 & 4.26 & 1.42 & .01 & 1.43 & .01 & .92 & .01 & 20.61 & .12 & 17 \\
\hline DM & 1188.84 & 9.71 & 435.28 & 11.20 & 249.44 & 10.99 & 1.42 & .01 & 1.43 & .01 & .93 & .00 & 20.54 & .23 & 8 \\
\hline UNOP & 1159.05 & 10.52 & 440.99 & 5.17 & 228.17 & 4.30 & 1.40 & .01 & 1.42 & .01 & .91 & .00 & 20.30 & .16 & 15 \\
\hline
\end{tabular}

Note-All brain weights are in milligrams; body weights are in grams. Linear measurements are in centimeters. $F B=$ forebrain; $H B=$ hindbrain; $L=$ length $W=$ width $D=$ depth. $A D X=$ adrenalectomized $; H A M=$ sham-adrenalectomized; $D M=$ demedullated; $U N O P$ = unoperated control. All ADX measurements except body length differ significantly (by at least $p<.05$ ) from those of other groups; these groups do not differ among themselves. The body weights of ADXs are significantly lighter than all other groups; DM body weights are significantly heavier than UNOPs and ADXs. 


\section{EXPERIMENT 2}

In view of the central role of the adrenal cortex in the modulation of brain size, replacement studies were undertaken in order to determine the glucocorticoid whose administration would return brain weights to SHAM levels. The adrenal cortex secretes hormones of two broad types, mineralocorticoids and glucocorticoids. In this experiment, we examined the contribution of mineralocorticoids in a dose-response design.

\section{Method}

Adrenalectomized female rats were prepared exactly as in the previous experiment. Deoxycorticosterone (DOC) was administered daily, beginning 2 days after surgery (at age 27 days) at three doses: 0 (vehicle), 1 , and $3 \mathrm{mg} / \mathrm{kg}$ in sesame oil $(\mathrm{n}=6)$. The concentrations were adjusted periodically in order that injection volumes be between .1-.2 ml. Aldosterone (ALDO) was prepared similarly and administered daily at doses of $0, .02$, and $.5 \mathrm{mg} / \mathrm{kg}(\mathrm{n}=6)$. Hormones were injected until autopsy at 65 days of age. These doses were selected with an eye toward approximating physiological levels (Gray, 1976) with the middle doses and exaggerating the extremes with the zero and high doses. Brain and body measurements were taken as before.

\section{Results and Discussion}

Neither DOC nor ALDO at any dose significantly decreased brain weights or dimensions from those of the adrenalectomized condition $[F s(2,15)=.04-2.18$, ps $>.15-.95$. Forebrain weights ranged from $1,205.8$ to $1,284.8,1,323.9$ to $1,381.2,1,236.5$ to $1,385.1$, and $1,303.7$ to $1,399.3 \mathrm{mg}$ for DOC low- and highand ALDO low- and high-dose groups, respectively. The range for ADXs was $1,249.6$ to $1,396.0 \mathrm{mg}$. All other brain measures showed a similar overlap. What was interesting, however, was that the body-weight loss of ADX subjects reported in the previous experiment was reversed by the mineralocorticoid replacement. From a mean of $205.1 \mathrm{~g}$ for ADXs, DOC at 1 and $3 \mathrm{mg}$ each significantly raised respective body weights to 244.1 and $264.7 \mathrm{~g}[\mathrm{~F}(2,15)=16.41$, $\mathrm{p}<$ .0002 ]. The effects of these two doses of DOC did not differ significantly from each other.

Aldosterone at .02 and $.5 \mathrm{mg} / \mathrm{kg}$ resulted in bodyweight increases to 226.8 and 253.2 , respectively $[F(2,15)=4.55, p<.029]$, values which differed significantly from each other as well as from those of ADXs. This body-weight difference was accomplished without significant changes in body length. Obvious visual differences in abdominal body fat suggested that the weight increases were due, at least in part, to an increased deposition of fatty tissue.

Brain weight and body weight, although statistically independent in the present experiment, are not necessarily conceptually independent. Frequently, brain weight is considered in some proportion to body weight (Wimer, 1979, pp. 148-163). In this way, true advances in brain growth across development or evolution can be observed apart from the obligatory relationship the size of all organs hold with respect to overall body size changes. Casting the data into a proportion (brain weight:body weight), mineralocorticoids would seem to diminish relative brain size. Thus, those who hold this ratio to be critical, as we once did (Devenport, 1979), will regard DOC and ALDO as relevant to the regulation of brain size. However, we can now more confidently attribute the somatic effects of mineralocorticoids to fat deposition (Devenport, Murray, \& Torres, 1982) and not skeletal length. In view of this, we reverse our earlier opinion and conclude that mineralocorticoids affect body, but not brain, size. Experiment 3 offers further compelling justification for this position, and the issue will be taken up there.

\section{EXPERIMENT 3}

\section{Method}

Corticosterone, the rat's chief glucocorticoid, was replaced on a daily basis in adrenalectomized rats at doses of 0 (vehicle), 1 , 3 , and $15 \mathrm{mg} / \mathrm{kg}$ (see Table 2 for listing of groups and $\mathrm{ns}$ ); these hormones were administered to rats of the same age, sex, origin, and surgery as those of the previous experiments. The hormones were administered across the same time span (Days 27-65). Subjects in the 0-dose group were the same as those of Experiment 1. Autopsy measurements were made as before.

\section{Results and Discussion}

Corticosterone exerted a clear suppressive influence on all brain measurements $[\mathrm{Fs}(3,35)=8.07$ 18.82 , ps $<.0001-.0003$ ], and the suppression was generally dose-dependent. For example, the forebrains of both the $3-$ and $15-\mathrm{mg} / \mathrm{kg}$ dose groups weighed significantly less than those of the 0 - and $1-\mathrm{mg} / \mathrm{kg}$ groups (Table 2). And the $15-\mathrm{mg} / \mathrm{kg}$ dose significantly diminished forebrain size beyond that of the $3-\mathrm{mg} / \mathrm{kg}$ dose. The same pattern of results was obtained for forebrain width. In other instances, the same trend was present, but only the effects of the highest corticosterone dose differed reliably from those of the other groups.

Corticosterone obviously decreased brain growth, but it just as plainly decreased body growth. The dose which returned forebrain weight to that of SHAMs (Experiment 1) also suppresses body weight. This leaves us in the position of supporting a contradiction: that higher doses of corticosterone increase brain growth and that they dramatically reduce it. The paradox turns on the question of the proper index of brain size: brain:body ratios (as may be calculated from Table 2) are increased by corticosterone administration, but absolute brain weights are diminished. However, taking stock of the initial question-what hormonal absences are responsible for the ADX's unequivocal brain enlargement and body-weight loss-the answer seems plain and, if correct, obviates the problem of brain-growth indices. Since mineralocorticoids (among other things) pro- 
Table 2

Effects of Daily Corticosterone Administration on Mean Brain and Body Size (Days 27-65)

\begin{tabular}{|c|c|c|c|c|c|c|c|c|c|c|c|c|c|c|c|}
\hline \multirow[b]{3}{*}{ Dose } & \multicolumn{6}{|c|}{ Weight } & \multicolumn{8}{|c|}{ Linear Dimensions } & \multirow[b]{3}{*}{$\mathrm{n}$} \\
\hline & \multicolumn{2}{|c|}{ FB } & \multicolumn{2}{|c|}{ HB } & \multicolumn{2}{|c|}{ Body } & \multicolumn{2}{|c|}{ FB-L } & \multicolumn{2}{|c|}{ FB-W } & \multicolumn{2}{|c|}{ FB-D } & \multicolumn{2}{|c|}{ Body } & \\
\hline & Mean & SEM & Mean & SEM & Mean & SEM & Mean & SEM & Mean & SEM & Mean & SEM & Mean & SEM & \\
\hline 0 & 1302.86 & 14.42 & 478.16 & 8.47 & 205.14 & 4.39 & 1.46 & .01 & 1.46 & .01 & .96 & .01 & 20.18 & .21 & 20 \\
\hline 1 & 1282.02 & 20.75 & 466.33 & 8.32 & 232.67 & $11.71 *$ & 1.46 & .01 & 1.43 & $.01 *$ & .96 & .01 & 20.67 & .22 & 6 \\
\hline 3 & 1214.13 & $21.46 *$ & 456.04 & 11.50 & 221.71 & 5.41 & 1.42 & .01 & 1.45 & .01 & .96 & .01 & 20.64 & .16 & 7 \\
\hline 15 & 1084.53 & $33.94 *$ & 403.30 & $9.95^{*}$ & 155.27 & $4.01 *$ & 1.37 & $.02^{*}$ & 1.39 & $.01 *$ & .89 & $.02 *$ & $* *$ & $* *$ & 6 \\
\hline
\end{tabular}

Note-All brain weights are in milligrams; body weights are in grams. Linear measurements are in centimeters. FB = forebrain; HB = hindbrain; $L=$ length,$W=$ width; $D=$ depth. Dose = corticosterone dose (in milligrams per kilogram). ${ }^{*}$ Differs significantly from 0 -dose group by at least $p<.05$. ${ }^{* *}$ These data were inadvertently lost.

mote body-weight gain while affecting brain size not at all, their absence is quite likely responsible for the smaller body size of adrenalectomized animals. Corticosterone reduces brain size and, at the dose required to replicate the SHAM brain size, probably results in a loss of body weight, if influencing the latter at all, its absence having opposite effects. A combined dose of corticosterone and DOC administered to adrenalectomized subjects should produce the smaller brain but larger body characteristics of SHAMs relative to ADXs. Experiment 4 examines this question.

\section{EXPERIMENT 4}

Having tried several dose combinations not to be reported, this experiment is meant to stand as a demonstration that a particular combination of corticosterone and DOC is capable of closely restoring, in most respects, the body and brain characteristics of SHAM-operated rats, that is, those with adrenals intact.

\section{Method}

Adrenalectomized rats were prepared as before. For their 38 days of treatment, a combined dose of $8 \mathrm{mg} / \mathrm{kg}$ corticosterone and $.6 \mathrm{mg} / \mathrm{kg} \mathrm{DOC}$ was administered subcutaneously in sesame oil $(C+D n=9)$. For comparison, other subjects were adrenalectomized (ADX $n=10)$ or sham-operated (SHAM $n=10)$ and received daily vehicle injections. Brain and body weights and di- mensions were taken as previously described. Blood serum was collected, and titers of corticosterone were determined by radioimmunoassay.

\section{Results and Discussion}

Table 3 details the outcome of the combined adrenal steroid replacement regimen across all dependent measures. For the most part, we were able to meet our goal of restoring the characteristics of SHAMs to adrenalectomized rats by administering the combination dose. Mean micrograms corticosterone per $100 \mathrm{ml}$ were boosted from $1.37 \pm .56$ for the nonreplacement (ADX) group to $18.17 \pm 6.58$ for $\mathrm{C}+\mathrm{Ds}$. Although this was substantially lower than the $45.73 \pm 4.92 \mathrm{mg} \%$ corticosterone titer for SHAMs, the latter value was exaggerated because of the stress of sacrifice procedures. The $C+D$ values compare quite favorably with nonstressed subjects reported in the literature. Although our samples yielded corticosterone titers that were somewhat low for female rats, the samples were taken $18 \mathrm{~h}$ postinjection. In fact, our corticosterone dose was probably higher than necessary.

Groups differed in forebrain $[F(2,26)=22.53$, $\mathrm{p}<.0001]$, hindbrain $[\mathrm{F}(2,26)=13.36, \mathrm{p}<.0001]$, and body weight $[F(2,26)=13.57, p<.0001]$. Individual comparisons $(\alpha=.05)$ found these differences to be attributable to the significantly lighter portions of brain in C+Ds and SHAMs. In contrast, body

Table 3

Effect of Daily Hormonal Injections (Days 27-65) on Mean Growth Measurements

\begin{tabular}{|c|c|c|c|c|c|c|c|c|c|c|c|c|c|c|c|}
\hline \multirow[b]{3}{*}{ Group } & \multicolumn{6}{|c|}{ Weight } & \multicolumn{8}{|c|}{ Linear Dimensions } & \multirow[b]{3}{*}{$\mathbf{n}$} \\
\hline & \multicolumn{2}{|c|}{ FB } & \multicolumn{2}{|c|}{ HB } & \multicolumn{2}{|c|}{ Body } & \multicolumn{2}{|c|}{ FB-L } & \multicolumn{2}{|c|}{ FB-W } & \multicolumn{2}{|c|}{ FB-D } & \multicolumn{2}{|c|}{ Body } & \\
\hline & Mean & SEM & Mean & SEM & Mean & SEM & Mean & SEM & Mean & SEM & Mean & SEM & Mean & SEM & \\
\hline ADX & 1317.29 & 24.45 & 485.65 & 7.79 & 198.90 & 5.26 & 1.48 & .01 & 1.47 & .01 & .97 & .01 & 20.18 & .21 & 10 \\
\hline$C+D$ & 1163.48 & 12.47 & 436.77 & 8.47 & 220.89 & 4.81 & 1.42 & .01 & 1.40 & .01 & .92 & .02 & 20.13 & .24 & 9 \\
\hline SHAM & 1168.12 & 15.51 & 437.14 & 7.06 & 240.00 & 6.61 & 1.42 & .01 & 1.43 & .01 & .92 & .01 & 20.65 & .16 & 10 \\
\hline
\end{tabular}

Note-All brain weights are in milligrams; body weights are in grams. Linear measurements are in centimeters. $F B=$ forebrain; $H B=$ hindbrain; $L=$ length $W=$ width $D=$ depth. $A D X=$ adrenalectomized $C+D=$ corticosterone, $8 \mathrm{mg} / \mathrm{kg} ; \quad D O C, .6 \mathrm{mg} / \mathrm{kg} ; \mathrm{SH} A M=$ sham-adrenalectomized. ADX and SHAM groups received equivalent amounts of the oil vehicle. All groups' measurements except body length differ significantly by at least $p<.05$ from ADXs. The other groups differ only in body weight. 
weight was elevated beyond ADXs in these two groups.

As for linear dimensions, length $[F(2,26)=6.08$, $\mathrm{p}<.007]$, width $[\mathrm{F}(2,26)=9.01, \mathrm{p}<.001]$, and depth $[F(2,26)=5.08, p<.014]$ differences were obtained. No significant differences were found in body length $[F(2,26)=2.12, p>.05]$. As expected, $C+D s$ and SHAMs paralleled each other in their significant departure from ADXs on each brain dimension.

This close resemblance of C+Ds to SHAMs leads us to conclude that our replacement dosage approximated endogenous levels. Yet the regimen was not perfect: SHAMs and C+Ds differed on one score, body weight. Both were heavier than ADXs, but SHAMs also weighed significantly more than $C+D s$. This, taken together with the trend in C+Ds toward brains that were smaller than those of SHAMs, suggests that the corticosterone part of the dose should be decreased and the DOC part increased. Rather than the 8:.6 ratio used in this experiment, we now (Devenport \& Devenport, Note 1) employ a 7:1 combination that seems quite accurate.

\section{GENERAL DISCUSSION}

We can fairly confidently conclude that corticosterone is the active adrenal factor whose removal by adrenalectomy is responsible for the enhanced brain growth previously reported (Devenport, 1979; Meyer, 1981). We also now report that the brain growth is general, involving hindbrain and all forebrain dimensions. The chief cause of ADX weight loss is mineralocorticoid deficiency. This latter finding is intriguing as aldosterone and deoxycorticosterone are rarely considered apart from their role in salt metabolism and associated body fluid dynamics. This is the case despite occasional hints (e.g., Gray, 1976) in the literature that they may be involved in the control of body size. The present studies found body weight to be reliably increased as a consequence of DOC or ALDO administration. In the face of unchanged skeletal length, and in view of our impression from autopsy, the mineralocorticoids play a role in the control of body fat metabolism.

In specifying the hormonal combination that produces the brain/body characteristics of intact animals, we have an additional goal. Use of this combination imparts a degree of experimental control that is otherwise absent. Although adrenals may be removed, they cannot be systematically replaced. With hormonal injections, such questions as whether adrenalectomy-induced brain changes are reversible, have "sensitive" periods, or have nonresponsive periods, etc., can be investigated.

Indeed, virtually nothing is known about the functional consequences of brain size differences brought about by hormonal manipulation. Perhaps it is similar to, or the same as, "enrichment"-induced brain expansion (Rosenzweig, Bennett, \& Krech, 1964). A recent review has concluded that the hormonal mediation of this effect is a distinct possibility (Uphouse, 1980). And the underlying cellular changes responsible for the enlargement are not known as yet. In any case, much interest attaches to the molar issue of brain size per se from an evolutionary (Jerison, 1979, pp. 30-60) and genetic (Roderick, 1979, pp. 133-147) perspective to the clinical level, where schizophrenia (Weinberger \& Wyatt, 1980, pp. 29-41), chronic alcoholism (Carlen, Wilkinson, Holgate, \& Wortzman, 1979; Golden, Graber, Blose, Berg, Coffman, \& Bloch, 1981), and other disorders (e.g., iatrogenic: Lagenstein, Willig, \& Kühne, 1979) are commonly characterized by smaller-than-normal brains, wider ventricles, and narrower gyri. Any set of naturally occurring hormones that are shown to alter brain size are therefore of considerable importance.

\section{REFERENCE NOTE}

1. Devenport, L. D., \& Devenport, J. A. Adrenocortical hormones and brain growth: Reversibility and differential sensitivity during development. Manuscript submitted for publication, 1982.

\section{REFERENCES}

Bohn, M. C., \& Lauder, J. M. The effects of neonatal hydrocortisone on rat cerebellar development. Developmental Neuroscience, 1978, 1, 250-266.

Bondonna, T. J., Jacquet, Y., \& Wolf, G. Perfusion-fixation procedure for immediate histological processing of brain tissue. Physiology \& Behavior, 1977, 19, 345-347.

Carlen, P. L., Wilkinson, D. A., Holgate, R., \& Wortzman, G. Computed tomography scans of alcoholics: Cerebral atrophy? Science, 1979, 204, 1237-1238.

Devenport, L. D. Aversion to a palatable saline solution in rats: Interactions of physiology and experience. Journal of Comparative and Physiological Psychology, 1973, 83, 98-105.

DEVENPORT, L. D. Adrenal modulation of brain size in adult rats. Behavioral and Neural Biology, 1979, 27, 218-221.

Devenport, L. D., Murray, C. G., \& Torres, A. Hormonal acceleration of fat deposition and secondary hyperphagia in rats. Neuroscience Abstracts, 1982, 8, 904.

Di Giusto, E. L., Cairncross, K., \& Kina, M. G. Hormonal influences on fear-motivated responses. Psychological Bulletin, $1971,75,432-444$.

Golden, C. J., Graber, B., Blose, I., Berg, R., Coffman, J., \& BLOCH, S. Difference in brain densities between chronic alcoholic and normal control patients. Science, 1981, 211, 508-510.

Gray, P. Effect of prestimulation on avoidance responding in rats, and hormonal dependence of the effect. Journal of Comparative and Physiological Psychology, 1976, 90, 1-17.

Jerison, H. J. The evolution of diversity in brain size. In M. E. Hahn, C. Jensen, \& B. C. Dudek (Eds.), Development and evolution of brain size. New York: Academic Press, 1979.

Lagenstein, I., Willia, R. P., \& Kühne, D. Cranial computed tomography (CCT) findings in children treated with ACTH and dexamethasone: First results. Neuropädiatrie, 1979, 10, 370-384.

Landfield, P. W., Baskin, R. K., \& Pither, T. A. Brain aging correlates: Retardation by hormonal-pharmacological treatments. Science, 1981, 214, 581-584.

Landfield, P. W., W AYmire, J. C., \& LYnCh, G. Hippocampal aging and adrenocorticoids: Quantitative correlations. Science, 1978, 202, 1098-1102. 
MEYER, J. Early adrenalectomy stimulates subsequent growth and development of the rat forebrain. Neuroscience Abstracts, 1981, 7, 289.

Pfaff, D. W., Silva, M. T. A., \& Weiss, J. M. Telemetered recording of hormone effects on hippocampal neurons. Science, $1971,172,394$.

Quarton, G. C., Clark, L. D., Cobb, S., \& Bauer, W. Mental disturbances associated with ACTH and cortisone: A review of explanatory hypotheses. Medicine, 1955, 34, 13-50.

RoDerick, T. H. Genetic techniques as tools for analysis of brainbehavior relationships. In M. E. Hahn, C. Jensen, \& B. C. Dudek (Eds.), Development and evolution of brain size. New York: Academic Press, 1979.

Rosenzweig, M. R., Bennett, E. L., \& Krech, D. Cerebral effects of environmental complexity and training among adult rats. Journal of Comparative and Physiological Psychology, $1964,80,304-313$.
Scheff, S. W., Bernardo, L. S., \& Cotman, C. W. Hydrocortisone administration retards axon sprouting in the rat dentate gyrus. Experimental Neurology, 1980, 68, 195-201.

UpHOUSE, L. Reevaluation of mechanisms that mediate brain differences between enriched and impoverished animals. Psychological Bulletin, 1980, 88, 215-232.

Weinberaer, D. R., \& WYATt, R. J. Structural brain abnormalities in chronic schizophrenia: Computed tomography findings. In C. F. Baxter \& T. Melnechuk (Eds.), Perspectives in schizophrenia research. New York: Raven, 1980.

Wimer, C. Correlates of mouse brain weight: A search for component morphological traits. In M. E. Hahn, C. Jensen, \& B. C. Dudek (Eds.), Development and evolution of brain size. New York: Academic Press, 1979.

(Manuscript received October 14, 1982; revision accepted for publication January 3, 1983.) 